\title{
Atividades Avançadas de Vida Diária (AAVD) e desempenho cognitivo entre idosos
}

\author{
Eduardo Moreira de Oliveira - Universidade de São Paulo, São Paulo, Brasil \\ Henrique Salmazo da Silva - Universidade de São Paulo, São Paulo, Brasil \\ Andrea Lopes - Universidade de São Paulo, São Paulo, Brasil \\ Meire Cachioni - Universidade de São Paulo, São Paulo, Brasil \\ Deusivania Vieira da Silva Falcão - Universidade de São Paulo, São Paulo, Brasil \\ Samila Satler Tavares Batistoni - Universidade de São Paulo, São Paulo, Brasil \\ Anita Liberalesso Neri - Universidade Estadual de Campinas, Campinas, Brasil \\ Mônica Sanches Yassuda - Universidade de São Paulo, São Paulo, Brasil
}

\begin{abstract}
Resumo
O estudo objetivou investigar a associação entre Atividades avançadas de vida diária (AAVD) e o desempenho cognitivo entre idosos participantes do estudo FIBRA em Ermelino Matarazzo, São Paulo. Foram investigados 302 idosos sem declínio cognitivo, classificados em muito ativos e pouco ativos nas AAVDs, e avaliados pelo Mini exame do estado mental (MEEM), bateria breve de rastreio cognitivo (BBRC), fluência verbal (FV), teste do desenho do relógio (TDR) e escala de depressão geriátrica (EDG). As AAVDs associaram-se ao desempenho no MEEM e no TDR, nas análises univariadas, mas esta associação perdeu significância no modelo multivariado. O desempenho, no MEEM, esteve associado à idade, escolaridade, sexo e número de sintomas depressivos. O escore, no TDR, associou-se à escolaridade. Conclui-se que a relação entre as AAVDs e o desempenho cognitivo parece ser modulada por variáveis sociodemográficas e por sintomas depressivos.
\end{abstract}

Palavras-chave: idoso; cognição; lazer.

Advanced Activities of Daily Living (AADL) and cognitive performance among older adults

\begin{abstract}
This study aimed to investigate the association between advanced activities of daily living (AADL) and cognitive performance in a community of dwelling older adults participants of the FIBRA study in Ermelino Matarazzo, São Paulo. 302 older adults without cognitive decline, classified as very active or little active in AADLs, were assessed by the Mini-Mental State Examination (MMSE), Brief Cognitive Screening Battery (BCSB), Verbal Fluency (VF), Clock Drawing Test (CDT), and Geriatric Depression Scale (GDS). The AADLs were associated with performance in the MMSE and CDT in univariate analyses, but the association lost statistical significance in the multivariate model. The MMSE score was associated with age, education, sex and depression symptoms. The CDT score was associated with education. The association between AADLs and cognitive performance seems to be modulated by socio-demographic variables and the number of depression symptoms.

Keywords: older adults; cognition; leisure.
\end{abstract}

\section{Actividades Avanzadas de la Vida Diaria (AAVD) y desempeño cognitivo entre ancianos}

\section{Resumen}

Este estudio investigó la asociación entre las Actividades Avanzadas de la Vida Diaria (AAVD) y el desempeño cognitivo entre las personas ancianas, participantes del Estudio FIBRA, en Ermelino Matarazzo, San Pablo. Fueron investigadas 302 ancianos, sin deterioro cognitivo, clasificados en muy activos y poco activos en las AAVD, y evaluadas por el Mini-Examen del Estado Mental (MEEM), la Batería Breve de Rastreo Cognitivo (BBRC), Fluidez Verbal (FV), Test del Dibujo del Reloj (TDR), y la Escala de Depresión Geriátrica (EDG). Las AAVD se asociaron con el rendimiento en el MEEM y en el TDR en los análisis univariados, pero perdieron importancia en el modelo multivariado. El desempeño en el MEEM estuvo asociado a la edad, escolaridad, sexo y número de síntomas depresivos. El resultado en el TDR se asoció a la escolaridad. La relación entre el rendimiento cognitivo y las AAVD parece estar regido por las variables sociodemográficas y por síntomas depresivos.

Palabras-clave: anciano; cognición; ocio.

\section{Introdução}

Existe grande variabilidade no envelhecimento cognitivo entre os indivíduos, dada à heterogeneidade de aspectos biológicos, socioeconômicos, culturais e ambientais que podem modular esse processo (Reuter-Lorenz, \& Park, 2010; Wilson et al., 2002).
Dessa forma, pesquisadores investigam a influência da participação social e do engajamento em atividades intelectuais e físicas na trajetória de envelhecimento cognitivo (Wang, Karp, Winblad, \& Fatiglioni, 2002; Vemuri et al., 2014).

As Atividades avançadas de vida diária (AAVDs) constituem um conjunto de atividades de lazer, 
realizadas no tempo livre, independentes do trabalho, que incluem o voluntariado, atividades educacionais, participação social na comunidade, sendo dependentes de motivação pessoal (Neri, 2005). As AAVDs podem ser divididas em físicas e sociais (Reuben, Laliberti, Hiris \& Mor, 1990) ou em sociais, produtivas, físicas e de lazer (Dias, 2009), sobrepondo-se conforme a finalidade e a natureza das atividades. Na literatura gerontológica, as AAVDs podem indicar boa saúde física e mental, e a redução no engajamento nessas atividades pode sugerir início de declínio funcional, alterações cognitivas e fragilidade (Dias, Duarte \& Lebrão, 2010; Kono, Kai, Sakato \& Rubenstein, 2007; Melo, 2009; Silva et al. 201).

As evidências indicam que idosos socialmente participativos e engajados em atividades de lazer tenderiam a ter melhor desempenho cognitivo (Figueiredo, Assis, Silva, Dias \& Mancini, 2013; Silva et al. 2014; Wang et al., 2002; Wang et al., 2012). Esses resultados indicam que a realização dessas atividades poderia ser recomendada como uma estratégia de prevenção para postergar o declínio cognitivo e possível início de síndromes demenciais (Vemuri et al., 2014; Wang et al., 2012).

Em estudo recente, Vemuri et al. (2014) observaram que idosos que possuíam o gene APOE4 e que encontravam-se no $75^{\circ}$ percentil de enriquecimento intelectual (maior escolaridade, ocupação estimulante ao longo da vida e maiores índices de participação em atividades na velhice) postergaram em 8,7 anos o aparecimento de declínio cognitivo em comparação aos idosos com baixo enriquecimento. Dessa forma, alguns pesquisadores defendem que a exposição do indivíduo a atividades complexas ao longo da vida pode estar associada à formação de reserva cerebral e cognitiva (Fratiglioni, Paillard-Borg, \& Winblad, 2004; Scarmeas et al., 2001; Stern, 2009).

Di Rienzo (2009), em estudo epidemiológico realizado no município de São Paulo, avaliou a participação de 1.520 idosos em atividades sociais, físicas, cognitivas e cotidianas e a relação com o desempenho cognitivo. Após 24 meses, 1.243 idosos foram reavaliados. Os resultados revelaram uma associação positiva entre a participação em atividades e o funcionamento cognitivo. Tal participação esteve associada à redução no risco para o comprometimento cognitivo amnésico e para demência, sugerindo que a realização dessas atividades pode contribuir com a preservação da cognição.

Embora o conhecimento acumulado permita inferir que as atividades estabelecem associações com a trajetória de desenvolvimento cognitivo na velhice (Hertzog, Kramer, Wilson, \& Lindenberger, 2009), o modo como essas variáveis interagem gera debates. Uma das questões colocadas é se haveria uma relação de causa e efeito entre a realização de AAVDs e desempenho cognitivo e os fatores predisponentes para a participação. Pergunta-se se os idosos que se engajaram em atividades cognitivamente complexas teriam maior reserva cognitiva ou cerebral ou se demonstram ganhos advindos da participação mesmo com baixa reserva cognitiva (Hertzog et al., 2009; Silva et al. 201; Silva \& Yassuda, 2013).

Teóricos, como Rowe \& Kahn (1998) propuseram que o engajamento com a vida é um dos aspectos essenciais do envelhecimento bem-sucedido, aliando-se ao adiamento da instalação das doenças crônicas e ao elevado desempenho físico e cognitivo. Salthouse (1991), por sua vez, propôs que idosos expostos a ambientes menos estimulantes reduziriam o uso de suas habilidades cognitivas, o que os levaria a um padrão de desempenho cada vez pior e, consequentemente, ao desuso de certas habilidades.

Contudo, o afastamento de algumas atividades pode ser subjacente a processos motivacionais e cognitivos complexos, envolvendo fatores como seleção e avaliação de custos e benefícios, tanto cognitivos quanto afetivos e emocionais, aliando-se ao significado que as atividades possuem para os idosos (Hess, 2014). De acordo com Hess (2014), quanto maior o custo em participar de uma atividade, menor o engajamento. $\mathrm{O}$ custo seria mensurado tanto por medidas fisiológicas (glicose, batimento cardíaco, funcionamento sensorial e cortical) quanto psicológicas (sensação de fadiga, atitudes, crenças, humor), aliando-se à avaliação dos resultados e consequências das atividades. Deve-se ressaltar ainda que certas atividades de lazer são frequentadas majoritariamente por idosos com maior renda, escolaridade e acesso, sendo que essas variáveis também impactam a cognição na velhice (Silva \& Yassuda, 2013; Zaitune et al., 2010).

A relação entre a realização de AAVDs e o desempenho cognitivo entre idosos brasileiros encontra-se relativamente pouco explorada. As atividades de lazer representam um campo importante para ações preventivas que podem ajudar a postergar o declínio cognitivo. Assim, o objetivo desse estudo foi verificar se a realização de AAVDs estaria associada ao melhor desempenho cognitivo entre idosos, após ajustes de variáveis moderadoras de natureza sociodemográfica e de humor. 


\section{Método}

\section{Participantes}

Foram utilizados os dados obtidos pela pesquisa Perfis de fragilidade em idosos brasileiros, realizada pela Rede FIBRA. Essa é uma rede de pesquisa de caráter multicêntrico e multidisciplinar que investigou as características, a prevalência e os fatores de risco relacionados à síndrome da fragilidade em idosos brasileiros. Fragilidade caracteriza-se como uma síndrome clínica que possui como fenótipo perda de massa muscular, lentidão na marcha, pouca força manual, autorrelato de fadiga e redução nas atividades realizadas. A síndrome da fragilidade encontra-se associada a desfechos negativos em saúde, como hospitalização e morte (Fried et al., 2001). A pesquisa realizada no distrito de Ermelino Matarazzo fez parte do polo coordenado pela UNICAMP, e as coletas ocorreram entre 2008 e 2009. Tratou-se de pesquisa de base domiciliar com recrutamento de participantes, realizado em duas ondas de arrolamento, em que os moradores do bairro que possuíam 65 anos ou mais, eram convidados a participar da pesquisa.

O distrito de Ermelino Matarazzo se localiza no extremo leste da cidade São Paulo, com população superior a 100.000 habitantes. Caracteriza-se como região de baixo nível socioeconômico num país em desenvolvimento ou de renda média. Segundo a Fundação Sistema Estadual de Análise de Dados (SEADE, 2010), apresenta área de $8,7 \mathrm{~km}^{2}$, subdividida em 143 setores censitários urbanos, com densidade populacional de $12.551,1$ habitantes $/ \mathrm{km}^{2}$. A população idosa $(\geq$ 65 anos) representa $4,5 \%$ dos habitantes do distrito.

A amostragem foi probabilística, por conglomerados, em dois estágios: setor censitário e domicílio. $\mathrm{O}$ método de sorteio das unidades primárias de amostragem (setores censitários) seguiu o mesmo padrão de todo o polo FIBRA UNICAMP. Para cálculo do tamanho da amostra no distrito de Ermelino Matarazzo, utilizou-se a expressão algébrica referente à estimação de proporções (Kish, 1965; Silva, 2001). O valor obtido, como tamanho mínimo da amostra, foi 384,16.

A amostra final de Ermelino Matarazzo ( $\mathrm{n}=384)$ foi composta, em sua maioria, por pessoas na faixa etária entre 65 e $69 \operatorname{anos}(38,0 \% ; \mathrm{M}=72,3 ; \mathrm{DP}=5,8)$, com predominância do sexo feminino $(67,2 \%)$. A maioria possuía o nível de escolaridade primário completo $(60,2 \% ; \mathrm{M}=3,4 ; \mathrm{DP}=2,8)$ e renda familiar de 1 a 3 salários mínimos $(53,8 \% ; \mathrm{M}=3,4 ; \mathrm{DP}=3,1)$. A amostra utilizada na presente análise foi composta por indivíduos que pontuaram acima da nota de corte do MEEM adotada no projeto FIBRA (17 pontos para analfabetos, 22 pontos para escolaridade entre 1 e 4 anos, 24 pontos entre 5 e 8 anos e 26 pontos para 9 e mais anos de escolaridade). Essasnotas foram calculadas a partir da média para cada faixa de escolaridade relatada em estudo anterior (Brucki, Nitrini, Caramelli, Bertolucci, \& Okamoto, 2003) menos um desvio padrão.

\section{Procedimentos}

Após sorteados os 66 setores censitários, os recrutadores (agentes comunitários de saúde e alunos de graduação em Gerontologia da Universidade de São Paulo) realizaram o arrolamento dos setores e localizaram as residências que continham moradores idosos. Estes foram, então, convidados a participar voluntariamente da pesquisa, recebendo um cartão com a data e local para a coleta de dados (uma igreja ou centro comunitário).

Foram utilizados os seguintes critérios de inclusão: ter idade igual ou superior a 65 anos, compreender as instruções, concordar em participar e ser residente permanente no domićlíio e no setor censitário. Os critérios de exclusão foram: 1. déficit cognitivo grave sugestivo de demência; 2 . uso de cadeira de rodas ou estar acamado; 3. ter sequelas graves de acidente vascular encefálico, com perda localizada de força e/ou afasia; 4. doença de Parkinson em estágio grave ou instável; 5. grave déficit de audição ou de visão, que dificultasse a comunicação; e 6 . doenças em estágio terminal. Os critérios de inclusão e de exclusão foram os utilizados no Cardiovascular Health Study e no Women's Health and Aging Studies, cujos dados foram usados para derivar o fenótipo de fragilidade, adotado pelo FIBRA (Ferrucci, Guralnik, Studenski, Fried, Cutler, \& Walston, 2004). A referida pesquisa foi submetida e aprovada pelo Comitê de Ética em Pesquisa UNICAMP.

\section{Instrumentos}

Para esta pesquisa, foram utilizados os dados de dois blocos de questões do protocolo da rede FIBRA polo UNICAMP. O primeiro utilizado foi o bloco $\mathrm{C}$ "status mental, autoavaliação da memória e rastreio cognitivo" do qual foram extraídos o Mini exame do estado mental (MEEM) (Brucki et al., 2003) e a Bateria breve de rastreio cognitivo (BBRC) (Vitiello, Ciríaco, Takahashi, Nitrini, \& Caramelli, 2007). O MEEM avalia, brevemente, sete aspectos da cognição (orientação temporal e espacial, memória imediata e tardia, atenção e cálculo, leitura, nomeação, praxia) e 
sua pontuação varia de 0 a 30 (Folstein, Folstein, \& McHugh, 1975). A BBRC avalia a nomeação, memória incidental, memória imediata, aprendizado e regate tardio para 10 figuras em preto e branco apresentadas em uma lâmina plastificada. Entre o aprendizado e o resgate tardio das figuras, foram realizados o teste de Fluência Verbal categoria animais (FV) e o Teste do Desenho do Relógio (TDR). Posteriormente, o idoso foi submetido à tarefa de reconhecimento das 10 figuras, quando se apresentou uma lâmina com 20 figuras, com as 10 figuras estudadas e 10 figuras distratoras. A BBRC gera escores que variam de 0 a 10 para a nomeação e cada tentativa de memorização das figuras. $\mathrm{O}$ escore de $\mathrm{FV}$ refere-se ao número de animais falados pelo participante em 60 segundos. O TDR foi avaliado pelos critérios de Shulman, Gold, Cohen e Zuchero (1993), que variam de 0 a 5 pontos. Para avaliação dos sintomas depressivos foi utilizada a Escala de Depressão Geriátrica (EDG) com 15 itens (Paradela, Lourenço, \& Veras, 2005).

Do bloco J sobre "capacidade funcional para AAVDs, AIVDs (Atividades instrumentais de vida diária) e ABVDs (Atividades básicas de vida diária) e expectativa de cuidado" foi utilizado o conjunto de questões relativas às AAVDs, quando o participante foi perguntado sobre a realização de atividades educacionais, cívicas, religiosas e de lazer. As respostas foram indicadas nas alternativas "nunca fez", "parou de fazer" e "ainda faz". Essas questões foram adaptadas de Baltes, Mayr, Borchelt, Maas e Wilms (1993); Reuben et al. (1990); Souza, Magalhães e Teixeira-Salmela (2006); e Strawbridge, Wallagen e Cohen (2002), e encontram-se no Anexo 1.

Para o presente estudo, foi calculado o número total de atividades que cada participante relatou fazer. Os participantes foram classificados em dois grupos: mais ativos, com maior participação nas AAVD, ou menos ativos, de acordo com sua pontuação nesse total. Foram considerados como mais ativos aqueles que realizavam quatro ou mais atividades e, como menos ativos, os idosos que relataram realizar três ou menos atividades, o que indicava estar no menor quartil da distribuição para essa variável. Estudos anteriores (Scarmeas et al., 2001) também dividiram a amostra entre idosos com alta e baixa participação em atividades de lazer (menos ou mais de seis atividades) a partir dos dados oriundos da população estudada, visto que não existe um consenso sobre a quantidade de atividades ou frequência/intensidade de participação que pudesse demarcar essas categorias.

\section{Análises estatísticas}

Foram realizadas análises descritivas para comparação do desempenho cognitivo e das características sociodemográficas entre idosos muito ativos e pouco ativos (variável categórica), utilizando-se o teste Mann-Whitney, devido à ausência de distribuição normal para as variáveis cognitivas. A associação entre AAVDs (variável ordinal -0 a 7 ) e as variáveis cognitivas e sociodemográficas foi analisada por modelo de regressão linear univariada e regressão linear múltipla. Foram consideradas como variáveis dependentes as variáveis de desempenho cognitivo (MEEM e TDR - para as quais foram identificadas diferenças significativas entre idosos muito ativos e pouco ativos) e, como variáveis independentes, a idade, escolaridade, sexo, pontuação na EDG, número de doenças e a pontuação nas AAVDs. Nas análises de regressão, optou-se por não incluir renda familiar para evitar efeito de colinearidade, devido à associação significativa entre escolaridade e renda. Ajustou-se a análise múltipla por variáveis que na análise univariada apresentaram valor de significância menor ou igual a 0,20. Para todas as análises, utilizou-se nível de significância de 5\% ( $<<0,05)$.

\section{Resultados}

A amostra de idosos em Ermelino Matarazzo foi composta por 384 idosos. Destes, 302 apresentaram pontuação acima da nota de corte no MEEM e compuseram a amostra para o presente estudo. A Tabela 1 apresenta as características sociodemográficas e clínicas da amostra. Na Tabela 2, encontram-se as comparações entre os idosos mais e menos ativos nas AAVDs. Observou-se que os idosos menos ativos eram mais velhos, apresentavam pior desempenho no MEEM e no TDR, bem como maior pontuação na EDG.

Aproximou-se da significância estatística a comparação entre a porcentagem de homens e mulheres e faixa de renda entre idosos menos e mais ativos. Houve tendência à significância, indicando maior número de homens e maior renda entre os mais ativos.

Para as variáveis MEEM e TDR, para as quais houve diferença significativa entre idosos mais e menos ativos, foram construídos modelos de regressão linear simples e múltipla. Conforme as Tabelas 4 e 5, foi observado que o número de AAVDs realizadas esteve associado com o escore do MEEM e do TDR, nas análises univariadas, com escores mais elevados entre os mais ativos, mas essa associação perdeu significância estatística após ajustes segundo idade, escolaridade, 
Tabela 1. Características Sociodemográficas e Clínicas da Amostra, Estudo FIBRA - Ermelino Mataraz:o, 2009

\begin{tabular}{lccccc}
\hline Variável & $\mathrm{N}$ & Média & D.P. & Mín & Máx \\
\hline Idade & 302 & 71,83 & 5,44 & 65 & 90 \\
Anos de escolaridade & 302 & 3,32 & 2,62 & 0 & 16 \\
Renda familiar (SM) & 257 & 3,33 & 2,95 & 0 & 24,10 \\
Número de doenças & 301 & 2,18 & 1,53 & 0 & 7 \\
EDG & 298 & 3,36 & 2,95 & 0 & 13 \\
MEEM & 302 & 24,90 & 2,82 & 17 & 30,0 \\
\hline
\end{tabular}

Nota. $\mathrm{SM}=$ salário mínimo, EDG=Escala de depressão geriátrica, MEEM=Mini exame do estado mental. Para renda familiar, alguns participantes optaram por não responder.

Tabela 2. Comparação entre Idosos mais Ativos e Menos Ativos nas AAVDs quanto às Características Sociodemográficas e Cognitivas, Estudo FIBRA - Ermelino Matarazzo, 2009

\begin{tabular}{lcccc}
\hline & Amostra total & Menos ativos & Mais ativos & p valor* \\
\hline Idade & $71,83(5,44)$ & $73,45(5,90)$ & $71,32(5,20)$ & 0,005 \\
Escolaridade & $3,32(2,62)$ & $2,93(2,37)$ & $3,45(2,68)$ & 0,104 \\
N $^{\circ}$ de doenças & $2,18(1,53)$ & $2,21(1,55)$ & $2,17(1,53)$ & 0,854 \\
MEEM & $24,90(2,82)$ & $24,01(3,09)$ & $25,21(2,65)$ & 0,003 \\
Memória tardia & $7,71(1,74)$ & $7,70(1,91)$ & $7,72(1,66)$ & 0,472 \\
FV & $12,31(3,48)$ & $11,93(3,51)$ & $12,44(3,47)$ & 0,540 \\
TDR & $2,78(1,65)$ & $2,30(1,68)$ & $2,93(1,61)$ & 0,01 \\
EDG & $3,36(2,56)$ & $4,04(2,70)$ & $3,15(2,49)$ & 0,007 \\
\hline
\end{tabular}

Nota. P valor refere-se ao teste Mann-Whitney, $\mathrm{p}<0,05$. MEEM=Mini exame do estado mental. FV=fluência verbal. TDR=Teste do desenho do relógio. EDG=Escala de depressão geriátrica.

Tabela 3. Comparação entre Idosos mais Ativos e Menos Ativos nas AAVDs quanto a Sexo e Renda. Estudo FIBRA-Ermelino Matarazzo, 2009

\begin{tabular}{lcccc}
\hline & & $\begin{array}{c}\text { Menos ativos } \\
\%(\mathrm{n})\end{array}$ & $\begin{array}{c}\text { Mais ativos } \\
\%(\mathrm{n})\end{array}$ & p valor* \\
\hline Sexo & Masculino & $33,33(42)$ & $66,67(84)$ & 0,064 \\
Faixa de renda & Feminino & $43,19(111)$ & $56,81(146)$ & \\
& 0 a $1 \mathrm{SM}$ & $45,81(71)$ & $54,19(84)$ & 0,058 \\
& $1,1-3 \mathrm{SM}$ & $41,61(67)$ & $58,39(94)$ & \\
$3,1-5 \mathrm{SM}$ & $21,43(9)$ & $78,57(33)$ & \\
& $5,1-10 \mathrm{SM}$ & $25(2)$ & $75(6)$ & \\
\hline
\end{tabular}

Nota. P valor refere-se ao teste Qui quadrado. $\mathrm{SM=}$ salários mínimos. 
Tabela 4. Modelos de Regressão Univariada com o Desempenho no MEEM e TDR como Variáveis Dependentes e Variáveis Sociodemográficas, Clínicas e Participação em AAVDs, como Variáveis Independentes,. Estudo FIBR A - Ermelino Mataražo, 2009

\begin{tabular}{lcccc}
\hline & \multicolumn{4}{c}{ Modelos univariados } \\
\cline { 2 - 5 } & $\begin{array}{c}\text { MEEM } \\
\text { Coef. (EP) }\end{array}$ & p-valor & $\begin{array}{c}\text { TDR } \\
\text { Coef. (EP) * }\end{array}$ & P-valor \\
\hline Idade & $-1,62(0,28)$ & 0,00 & $-0,00047(0,017)$ & 0,02 \\
Escolaridade & $0,55(0,05)$ & 0,00 & $0,244(0,034)$ & 0,00 \\
$\mathrm{~N}^{\circ}$ de doenças & $-0,221(0,105)$ & 0,36 & $-0,142(0,062)$ & 0,02 \\
EDG & $-0,268(0,62)$ & 0,00 & $-0,085(0,037)$ & 0,02 \\
AAVD & $0,184(0,74)$ & 0,01 & $0,104(0,043)$ & 0,02 \\
Sexo & $-0,83(0,31)$ & 0,01 & $-0,31(0,18)$ & 0,89 \\
\hline
\end{tabular}

Nota. $*$ Coeficiente $(\mathrm{EP}=$ erro padrão). EDG=Escala de depressão geriátrica. AAVD=Atividades avançadas de vida diária.

Tabela 5. Modelo de Regressão Linear Múltiplo para o Desempenho no MEEM e TDR como Variáveis Dependentes e Variáveis Sociodemográficas, Clínicas e Participação em AAVDs, como Variáveis Independentes. Estudo FIBRA - Ermelino Matarazzo, 2009

\begin{tabular}{lcccc}
\hline & \multicolumn{4}{c}{ Modelo múltiplo } \\
\cline { 2 - 5 } & MEEM & p-valor & TDR & p-valor \\
\hline Idade & $-1,27(0,25)$ & 0,00 & $0,0002(0,017)$ & 0,10 \\
Escolaridade & $0,48(0,54)$ & 0,00 & $0,219(0,035)$ & 0,00 \\
N $^{\circ}$ de doenças & $-0,005(0,89)$ & 0,53 & $-0,09(0,063)$ & 0,14 \\
EDG & $-0,144(0,55)$ & 0,01 & $-0,019(0,037)$ & 0,60 \\
AAVD & $-0,009(0,71)$ & 0,89 & $0,041(0,047)$ & 0,38 \\
Sexo & $-0,84(0,29)$ & 0,01 & $0,05(0,19)$ & 0,79 \\
Constante (B0) & $34,22(1,96)$ & 0,00 & $4,068(1,268)$ & 0,01 \\
\hline
\end{tabular}

Nota. $*$ Coeficiente $(\mathrm{EP}=$ erro padrão). EDG=Escala de depressão geriátrica. AAVD=Atividades avançadas de vida diária.

sexo, número de doenças e sintomas depressivos. $\mathrm{Na}$ modelagem múltipla, estiveram associados ao melhor desempenho no MEEM ter menor idade, escolaridade mais elevada, ser do sexo masculino e apresentar menor pontuação na EDG. Após ajustes, o escore no TDR apresentou associação significativa apenas com a escolaridade, indicando que os idosos com maior escolaridade apresentaram melhor desempenho nesse teste.

\section{Discussão}

O presente estudo objetivou investigar a relação entre a realização de AAVDs e o desempenho cognitivo em idosos residentes na comunidade, controlando-se o impacto de variáveis sociodemográficas e o número de sintomas depressivos, visto que são fatores que sabidamente influenciam a cognição. Nas análises de regressão simples, as AAVDs apresentaram associação com os escores do MEEM e o do TDR, mas a associação perdeu significância estatística após ajustes segundo idade, escolaridade, sexo, número de doenças e sintomas depressivos.

Embora diversos estudos tenham destacado a associação entre a realização de atividades e melhor desempenho cognitivo na velhice (Figueiredo et al., 2013; Hultsch, Hertzog, Small, \& Dixon, 1999; Vemuri et al., 2014; Wang et al., 2012), estudo anterior, em consonância com os dados apresentados, relatou que a relação entre atividades e cognição é moderada por condições socioeconômicas (Aartsen, Smits, van Tilburg, Knipscheer, \& Deeg, 2002). Os resultados observados sugerem que a associação entre cognição e a realização de atividades é complexa, sendo, possivelmente, mediada pela escolaridade, hábitos culturais, nível socioeconômico, 
saúde física, mobilidade, saúde emocional e motivação em desempenhar as atividades (Dias, 2009; Hess, 2014). Ademais, a motivação parece exercer um papel na seleção e engajamento nas atividades, envolvendo uma interação entre custos (fisiológicos, psicológicos), avaliação dos riscos associados à participação e significado pessoal em desempenhá-las (Hess, 2014).

No que se refere aos custos, o engajamento e a realização das AAVDs tende a declinar com o envelhecimento devido ao surgimento de problemas de saúde e barreiras sociais enfrentadas pelos idosos. Ashworth e Reuben (1994) mostraram que idosos desempenham menos AAVDs que jovens, por receio dos riscos associados a essas atividades. No presente estudo, embora a amostra tenha sido composta por indivíduos idosos com 65 anos ou mais, foi possível verificar um padrão semelhante a este, visto que os idosos menos ativos eram mais velhos. Na pesquisa FIBRA realizada no município de Campinas, encontrou-se que as AAVDs mais desempenhadas são aquelas que envolvem receber visitas e frequentar cultos, missas e encontros religiosos, ainda assim, com menor participação entre os mais longevos (Neri, Costa, Maríncolo, \& Ribeiro, 2011).

Kono et al. (2007) avaliaram 107 idosos e a relação entre ser frágil e a frequência de saídas de casa. Foi constatado que os idosos que saíam de casa mais de quatro vezes por semana tiveram suas AVDs preservadas por 22 meses, enquanto os indivíduos que saíam entre uma e três vezes apresentaram manutenção nas AVDs por apenas nove meses. Esses dados sugeriram que existe uma relação entre a realização de AAVDs e a independência na vida diária.

Além dos recursos físicos para desempenhar as atividades, fatores motivacionais teriam relação tanto com o desempenho cognitivo quanto com variáveis emocionais (Hess, 2014). Em consonância com o presente estudo, Isaak, Stewart, Artero, Ancelin e Ritchie (2009), após avaliarem 1.849 idosos, verificaram que quanto maior a frequência de realização de atividades sociais, menor a prevalência de sintomas depressivos. $\mathrm{Na}$ presente pesquisa, os idosos com menor participação em AAVDs, também, apresentaram maior número de sintomas depressivos. Esses achados sugerem que há um importante componente motivacional para a realização das AAVDs. Sabe-se, no entanto, que os sintomas depressivos influenciam negativamente a cognição de idosos (Plati, Covre, Lukasova, \& Macedo, 2006; Rook, Mavandadi, Sorkin, \& Zettel, 2007; Shimada et al., 2014), predizendo o declínio cognitivo (Paterniti, Verdier-Taillefer, Dufouil, \& Alpérovitch, 2002).
Em estudo recente, Shimada et al. (2014) observaram que idosos com sintomas depressivos, além de apresentaram pior desempenho cognitivo, manifestaram menor concentração sérica de Brain derived neurotrophic fator (BDNF) e maior atrofia no lobo temporal medial direito, quando comparados a idosos sem sintomas depressivos. Desse modo, os sintomas depressivos em idosos podem explicar menor engajamento em atividades complexas e pior desempenho cognitivo.

No presente estudo, a análise de regressão ajustada indicou a usual associação entre o escore no MEEM e idade, escolaridade e pontuação na EDG, e entre o TDR e a escolaridade. Esses resultados estão em consonância com a literatura, indicando que idosos mais jovens e com maior escolaridade tenderiam a apresentar melhor desempenho cognitivo (Silva et al., 2014).

Dentre as limitações do estudo, aponta-se a ausência do histórico de desempenho nas AAVDs, o que impossibilitou a identificação de idosos que estariam reduzindo a participação em atividades. Adicionalmente, o número de AAVDs realizadas pode não ser a maneira ideal de avaliar o grau de envolvimento com atividades. Frequência semanal ou mensal de engajamento nas atividades, número de horas de participação e o número de anos de realização das atividades poderiam ser informativos. Ademais, existem evidências de que o engajamento em AAVDs simbolizaria a disponibilidade de uma gama considerável de recursos cognitivos e/ou socioeconômicos presentes no curso de vida, na meia-idade e na velhice, possivelmente não avaliados na presente pesquisa (Kareholt, Lennartsson, Gatz, \& Parker, 2011; Wang et al., 2012). Quanto aos méritos do estudo, destaca-se o fato de ser de base populacional e ter incluído uma bateria cognitiva que avaliou aspectos diversos da cognição.

Destaca-se, ainda, que a ausência de um consenso sobre a melhor maneira de avaliar o engajamento em AAVDs representa um desafio nesse campo de investigação. Existe grande variabilidade no tipo de atividades associadas ao conceito de AAVD, o que dificulta a construção de uma escala de mensuração desse construto. Nesse campo, ainda não há um consenso quanto aos protocolos ideais de avaliação, terminologia e conjunto de atividades envolvidas, visto que esse é um construto recente (Dias, 2009).

\section{Conclusões e Considerações Finais}

Os dados do presente estudo indicaram que idosos com maior participação nas AAVDs apresentaram 
melhor desempenho no MEEM e TDR. Entretanto, os dados sugerem que a associação entre a realização de atividades e o desempenho cognitivo é modulada por variáveis sociodemográficas e sintomas depressivos. Os dados também sugerem que os idosos que se engajam nas AAVDs com maior frequência são aqueles com melhor desempenho cognitivo, maior escolaridade, mais jovens e com menor número de sintomas depressivos.

A contribuição da realização de AAVDs para a saúde mental na velhice deve ser alvo de novas investigações. Os próximos estudos devem aperfeiçoar as estratégias de avaliação das AAVDs na velhice, com propostas de instrumentos que contemplem simultaneamente o número, a frequência e a intensidade de participação em AAVDs. Novos estudos, também, devem investigar a relação entre AAVDs e cognição por meio de intervenções controladas, para que se possa avaliar o impacto da realização de AAVDs em comparação a um grupo que não as realiza. Esses estudos poderão fortalecer dados epidemiológicos recentes que sugerem que políticas públicas devem apoiar o engajamento de idosos em atividades significativas de lazer e participação social para a promoção da saúde geral e preservação da cognição na velhice.

\section{Referências}

Aartsen, M. J., Smits, C. H. M., van Tilburg, T., Knipscheer, K. C. P. M., \& Deeg, D. J. H. (2002). Activity in older adults: Cause or consequence of cognitive functioning? A longitudinal study on everyday activities and cognitive performance in older adults. Journal of Gerontology: Psychological Sciences, 57(2), 153-162.

Ashworth, J. B., \& Reuben, D. B. (1994). Functional profiles of healthy older persons. Age and ageing, 23(1), 34-39.

Baltes, M. M., Mayr, U., Borchelt, M., Maas, I., \& Wilms, H. U. (1993). Everyday competence in old and very old age: An interdisciplinary perspective. Ageing and Society, 13(4), 657-680.

Brucki, S. M. D., Nitrini, R., Caramelli, P., Bertolucci, P. H. F., \& Okamoto, I. H. (2003). Sugestões para o uso do Mini-Exame do Estado Mental no Brasil. Arquivos de Neuropsiquiatria, 61(3-B), 777-781.

Di Rienzo, V. D. (2009). Participação em atividades e funcionamento cognitivo: estudo de coorte com idosos residentes em área de baixa renda no município de São Paulo (Tese de doutorado não publicada). Faculdade de Medicina da Universidade de São Paulo, São Paulo, Brasil.

Dias, E. G. (2009). Atividades avançadas de vida diária no envelhecimento: Um estudo de revisão. (Dissertação de mestrado não publicada). Faculdade de Saúde Pública da Universidade de São Paulo, São Paulo, Brasil.

Dias, E. G., Duarte, Y. A. O., \& Lebrão, M. L. (2010). Efeitos longitudinais das atividades avançadas de vida diária em idosos: Implicações para a reabilitação gerontológica. O mundo da saúde, 34(2), 258-267.

Ferrucci, L., Guralnik, J. M., Studenski, S., Fried, L. P., Cutler, G. B., \& Walston, J. (2004). Designing randomized, controlled trials aimed at preventing or delaying functional decline and disability in frail, older persons: A consensus report. Journal of the American Geriatrics Society, 52(4), 625-634.

Folstein, M. F., Folstein, S. E, \& McHugh, P. R. (1975). A practical method for grading the cognitive state of patient for clinician. Journal of Psychiatric Research, 12, 189-198.

Fratiglioni, L., Paillard-Borg, S., \& Winblad, B. (2004). An active and socially integrated lifestyle late in life might protect against dementia. Lancet Neurology, 3(6), 343-353.

Fried, L. P., Tangen, M. P., Walston, J., Newman, A. B., Hirsch, C., Gottdiener, J., Seeman, T., Tracy, R., Kop, W. J., Burke, G., \& McBurnie, M. A.(2001). Frailty in older adults: Evidence for a phenotype. The Journals of Gerontology Series A: Biological Sciences and Medical Sciences, 56, 146-157.

Figueiredo, C. S., Assis, M. G., Silva, S. L. A., Dias, R. C., \& Mancini, M. C. (2013) Functional and cognitive changes in community-dwelling elderly: Longitudinal study. Brazilian Journal of Physical Therapy, 17(3), 297-306.

Hertzog, C., Kramer, A. R., Wilson, R. S., \& Lindenberger, U. (2009). Enrichment effects on adult cognitive development: Can the functional capacity of older adults be preserved and enhanced? Psychological Science in the Public Interest, 9(1), 1-20.

Hess, T.M. (2014). Selective engagement of cognitive resources: Motivational influences on older adult's cognitive functioning. Perspectives on Psychological Science, 9(4), 388-407

Psico-USF, Bragança Paulista, v. 20, n. 1, p. 109-120, jan./abr. 2015 
Hultsch, D. F., Hertzog, C., Small, B. J., \& Dixon, R. A. (1999). Use it or lose it: Engaged lifestyle as a buffer of cognitive decline in aging? Psychology and Aging, 14(2), 245-263.

Isaak, V., Stewart, R., Artero, S., Ancelin, M., \& Ritchie, K. (2009). Social activities and improvement in depressive symptoms in older people: A prospective community cohort study. American Journal of Geriatric Psycbiatry, 17(8), 688-696.

Kareholt, I., Lennartsson, C., Gatz, M., \& Parker, M. G. (2011). Baseline leisure activity and cognition more than two decades later. International Journal of Geriatric Psycbiatry, 26(1), 65-74.

Kish, L. (1965). Survey sampling. New York: John Wiley \& Sons.

Kono, A., Kai, I., Sakato, C., \& Rubenstein, L. Z. (2007). Frequency of going out doors predicts long range functional change among ambulatory frail elders living at home. Archives of Gerontology and Geriatrics, 45(3), 233-42.

Melo, D. M. (2009). Fragilidade, desempenho de atividades avancadas de vida diária e saúde percebida em idosos atendidos em ambulatório de geriatria. (Dissertação de mestrado não publicada). Faculdade de Educação da Universidade Estadual de Campinas, Campinas, Brasil.

Neri, A. L. (2005). Palavras-chave em gerontologia. Campinas, SP: Editora Alínea.

Neri, A. L., Costa, T.B., Maríncolo, J. C. S., \& Ribeiro, L. H. M. (2011). Atividade física, envolvimento social, produtividade e satisfação com a vida. Em A. L. Neri \& M. E. Guariento (Org), Fragilidade, saúde e bem-estar em idosos: Dados do estudo FIBRA Campinas. (pp. 75-99). Campinas, SP: Editora Alínea.

Neri, A. L., Yassuda, M. S., Araújo L. F., Eulálio, M. C., Cabral, B. E., Siqueira, M. E., Santos, G. A., \& Moura, J. G. (2013). Metodologia e perfil sociodemográfico, cognitivo e de fragilidade de idosos comunitários de sete cidades brasileiras: Estudo Fibra. Cadernos de Saúde Pública, 29(4), 778-92.

Paterniti, S., Verdier-Taillefer, M. H., Dufouil, C., \& Alpérovitch, A. (2002). Depressive symptoms and cognitive decline in elderly people: Longitudinal study. British Journal of psychiatry, 181, 406-410.

Paradela, E. M. P., Lourenço, R. A., \& Veras, R. P. (2005). Validação da escala de depressão geriátrica em um ambulatório geral. Revista de Saúde Pública, 39(6), 918-923.

Plati, M. C. F., Covre, P., Lukasova, K., \& Macedo, E. C. (2006). Depressive symptoms and cognitive performance of the elderly: Relationship between institutionalization and activity programs. Revista Brasileira de Psiquiatria, 28(2), 118-121.

Reuben, D. B., Laliberti, L., Hiris, J., \& Mor, V. (1990). A hierarchical exercise scale to measure function at the Advanced Activities of Daily Living (AADL) level. Journal of the American Geriatrics Society, 38(8), 855-861.

Reuter-Lorenz, P., \& Park, D.C. (2010). Human neuroscience and the aging mind: A new look at old problems. The Journal of Gerontology, 65(4), 405-415.

Rook, K. S., Mavandadi, S., Sorkin, D. H., \& Zettel, L. A. (2007) Optimizing social relationships as a resource for health and well-being in later life. Em C. M. Aldwin, C. L. Park, \& A. Spiro III (Ed.) Handbook of Health Psychology and Aging (p. 267-285). New York: The Guilford Press.

Rowe, J. W., \& Kahn, R. (1998). Successful aging. New York: Pantheon Books.

Salmazo-Silva, H., Duarte, Y. A. O., Andrade, F. B., Cerqueira, A. T. A. R., Santos, J. L. F., \& Lebrão, M. L. (2014). Correlates of above-average cognitive performance among older adults: the SABE study. Cadernos de Saúde Pública (ENSP. Impresso), 30, 1977-1986.

Salthouse, T. A. (1991). Theoretical perspectives on cognitive aging. Hillsdale, NJ: Erlbaum.

Scarmeas, N., Levy, G., Tang, M. X., Manly, J., \& Stern, Y. (2001). Influence of leisure activity on the incidence of Alzheimer's disease. Neurobiology, 57(12), 2236-2242.

SEADE - Fundação Sistema Estadual de Análise de Dados (2010). Município de São Paulo: População e estatísticas vitais. Disponível em: http://www. seade.gov.br/produtos $/ \mathrm{msp} /$ index.php?tip $=$ met 4 \&opt $=\mathrm{t} \&$ subtema $=$ null\&tema $=\mathrm{dem}$.

Shimada, H., Park, H., Makizako, H., Dot, T., Lee, S., \& Suzuki T. (2014) Depressive symptoms and cognitive performance in older adults. Journal of Psychiatric Research, 57,149-56.

Shulman, K., Gold, D. P., Cohen, C. A., \& Zuchero, C. A. (1993). Clock drawing and dementia in the 
community: A longitudinal study. International Journal of Geriatric Psycbiatry, 8(6), 487-496.

Silva, H. S. \& Yassuda, M.S. (2013) Engajamento social, lazer e envelhecimento cognitivo. Em Malloy-Diniz, L. F., Fuentes, D., \& Cosenza, R. M.. (Org.), Neuropsicologia do envelhecimento: Uma abordagem multidimensional (p. 428-437). Porto Alegre: Artmed.

Silva, N. N. (2001). Amostragem probabilística: Um curso introdutório. (2 ed.). São Paulo: EdUSP

Souza, A. C., Magalhães, L. C., \& Teixeira-Salmela, L. T. (2006) Adaptação transcultural e análise das propriedades psicométricas da versão brasileira do perfil de atividade humana. Cadernos de Saúde Pública, 22(12), 109-118.

Stern, Y. (2009). Cognitive reserve. Neuropsychologia, 47(10), 2015-2028.

Strawbridge, W. J., Wallagen, M. L., \& Cohen, R. (2002). Successful aging and well-being: Self-rated compared with Rowe e Kahn, The Gerontologist, 42(6), 727-883.

Vemuri, P., Lesnick, T. G., Przybelsi, S. A., Machulda, M., Knopman, D. S., Mielke, M. M., Roberts R. O., Geda Y. E., Rocca W. A., Petersen R. C., \& Jack Jr. C. R. (2014) Association of lifetime intellectual enrichment with cognitive decline in older population. JAMA Neurology, 71(8), 10-24

Vitiello, A. P., Ciríaco, J. G. M., Takahashi, D. Y., Nitrini, R., \& Caramelli, P. (2007). Avaliação cognitiva breve de pacientes atendidos em ambulatórios de neurologia geral. Arquivos de Neuro-Psiquiatria, 65(2A), 299-303.

Wang, H. X., Xu, W., \& Pei, J. J. (2012). Leisure activities, cognition and dementia. Biochimica et Biophysica Acta, 1822(3), 482-91.

Wang, H. W., Karp, A, Winblad, B., \& Fatiglioni, L. (2002). Late-life engagement in social and leisure activities is associated with a decreased risk of dementia: A longitudinal study from the Kungsholmen Project. American Journal of Epidemiology, 155(12), 1081-1087.

Wilson, R. S., Beckett, L. A., Barnes, L. L., Schneider, J. A., Bach, J., Evans, D. A., \& Bennett, D. A (2002). Individual differences in rates of change in cognitive abilities of older persons. Psychology of Aging, 17(2), 179-193.

Zaitune, M. P. A., Barros, M. B. A., César, C. L. G., Carandina, L., Goldbaum, M., \& Alves, M. C. G. P. (2010). Fatores associados à prática de atividade física global e de lazer em idosos: Inquérito de Saúde no Estado de São Paulo (ISA-SP), Brasil. Cadernos de Saúde Pública, 26(8), 1606-1618.

Recebido em: 10/02/2014

Primeira Reformulação em: 05/10/2014

Aprovado em: 10/12/2014 
Anexo 1

\begin{tabular}{lccc}
\hline Eu gostaria de saber qual é a sua relação com as seguintes atividades: & Nunca fez & Parou de fazer & Ainda faz \\
\hline 1. Fazer visitas na casa de outras pessoas. & 1 & 2 & 3 \\
2. Receber visitas em sua casa. & 1 & 2 & 3 \\
3. Ir à igreja ou templo para rituais religiosos ou atividades sociais & 1 & 2 & 3 \\
ligadas à religião. & & 3 \\
4. Participar de reuniões sociais, festas ou bailes. & 1 & 2 & 3 \\
5. Participar de eventos culturais, tais como concertos, espetáculos, & 1 & 2 & 3 \\
exposições, peças de teatro ou filmes no cinema. & 1 & 2 & 3 \\
6. Dirigir automóvel. & 1 & 2 & 3 \\
7. Fazer viagens de 1 dia para fora da cidade. & 1 & 2 & 3 \\
8. Fazer viagens de duração mais longa para fora da cidade ou do país. & 1 & 2 & 3 \\
9. Fazer trabalho voluntário. & 1 & 2 & 2 \\
10. Fazer trabalho remunerado. & 1 & & 3 \\
11. Participar de diretorias ou conselhos de associações, clubes, & & 3 \\
escolas, sindicatos, cooperativas ou centros de convivência, ou & & 3 \\
desenvolver atividades políticas. & & 3 \\
12. Participar de Universidade Aberta à Terceira Idade ou de algum \\
curso de atualização.
\end{tabular}

Nota. Protocolo de coleta de dados da Rede FIBRA pólo UNICAMP. 
Sobre os autores:

Eduardo Moreira de Oliveira é bacharel em Gerontologia, pela Escola de Artes Ciências e Humanidades - EACH USP, e especialista em Neurociências pela Faculdade de Medicina do ABC.

Henrique Salmazo da Silva é bacharel em Gerontologia, pela Escola de Artes, Ciências e Humanidades - EACH USP, e mestre em Ciências pela Faculdade de Saúde Pública, FSP USP.

Andrea Lopes é professora doutora em Gerontologia, pela Escola de Artes, Ciências e Humanidades, EACH USP.

Meire Cachionié professora associada em Gerontologia, pela Escola de Artes Ciências e Humanidades, EACH USP.

Deusivania Vieira da Silva Falcão é professora doutora em Gerontologia, pela Escola de Artes, Ciências e Humanidades, EACH USP.

Samila Satler Tavares Batistoni é professora doutora em Gerontologia, pela Escola de Artes, Ciências e Humanidades, EACH USP.

Anita Liberalesso Neri é professora titular da Faculdade de Educação, UNICAMP.

Mônica Sanches Yassuda é professora associada em Gerontologia, pela Escola de Artes Ciências e Humanidades, EACH USP.

Contato com os autores:

Mônica S. Yassuda - Gerontologia EACH USP

Av. Arlindo Bettio, 1000, sala 322-J - Ermelino Matarazzo, SP

CEP: 03828-000

E-mail: yassuda@usp.br 